\title{
Interferential Electrical Stimulation Versus Pulsed Electro-Magnetic Field in Management of Intermittent Claudication
}

Mona Mahmoud Mohamed ${ }^{* 1}$, Nesreen Gharib El-Nahas ${ }^{1}$, Mohamed Hamza Hamed ${ }^{2}$, Nagy Nassif Louis ${ }^{1}$

${ }^{1}$ Physical Therapy Department for Cardiovascular/Respiratory Disorder and Geriatrics, Faculty of Physical Therapy, Cairo University, Giza, Egypt.

${ }^{2}$ Department of Vascular Surgery, Faculty of Medicine, Al Azhar University, Egypt. *Corresponding Author: Mona Mahmoud Mohamed, Mobile: (+20)01127744248, E-Mail:dmonamahmoud925@gmail.com

\begin{abstract}
Background: Intermittent claudication is the occurrence of leg pain, aching, cramping, or fatigue triggered by walking and relieved by rest. It's a chronic disabling condition and the first symptom of peripheral arterial disease (PAD).

Objective: This study was designed to compare between the therapeutic efficacy of Interferential electrical stimulation and pulsed electromagnetic field (PEMF) in management of intermittent claudication (IC).

Subjects and methods: Forty five patients suffering from intermittent claudication due to atherosclerosis (Fontain stage II PAD) and randomly were assigned into three equal groups. Group "A" included 15 patients received 30 min of pulsed electromagnetic field in addition to heel raise exercise and calf stretch exercise and their medical treatment, group "B" included 15 patients received $20 \mathrm{~min}$ of interferential electrical stimulation in addition to heel raise exercise and calf stretch exercise and group " $\mathrm{C}$ " (control group) that included 15 patients received their medical treatment only. Ankle brachial pressure index, graded treadmill exercise testing to determine absolute claudication distance (ACD), Peak walking time (PWT) and walking impairment questionnaire (distance score, speed score, symptoms impairment score) were recorded pre and post the two months period for all groups.

Results: All measured parameters were significantly improved in all groups with the greatest improvement was in group (A) and the least improvement was in group (C) except for ankle brachial index that was changed significantly in group (A) only.

Conclusion: PEMF could be an effective vascular rehabilitation modality for improving walking efficiency and functional capacity in patients with intermittent claudication. It significantly increases the blood flow in peripheral arteries and so on can delay the complications of intermittent claudication.

Keywords: Pulsed electromagnetic field, Interferential electrical stimulation, Intermittent claudication, Peripheral artery disease.
\end{abstract}

\section{INTRODUCTION}

Peripheral arterial disease (PAD) is chronic arterial occlusive disease of lower extremities caused by atherosclerosis leading to an inadequate blood supply due to narrowing or hardening of the arteries ${ }^{(\mathbf{1})}$. About half of the patients with PAD are asymptomatic. From the other half, about $40-45 \%$ present with intermittent claudication ${ }^{(2)}$. Muscle ischemia during exercise and reperfusion after claudication limit exercise, which is associated with an increase in oxidant stress. The production of oxygen-free radicals may be a unifying mechanism of vascular and skeletal muscle injury in PAD ${ }^{(3)}$. Cigarette smoking is by far the most potent risk factor for development of peripheral atherosclerosis and intermittent claudication. Other major risk factors are increasing age, diabetes, hypertension, hyperlipidemia and hyperhomocysteinaenmia. In younger patients intermittent claudication may be caused by conditions such as popliteal artery entrapment, cystic adventitial disease, fibromuscular dysplasia and external compression syndromes ${ }^{(4)}$.

Interferential electrical stimulation uses a current in the mid-frequency range of $1-100 \mathrm{KHz}$. It is an amplitude-modulated alternative current produced by cross-interference of different currents within the body, thereby transmitting a burst frequency in the biological range into a continual flow of electric potential. Interferential stimulation stimulate arterial and capillary dilatation, improve tissue oxygenation and metabolism and decrease blood viscosity in the region applied. The frequency rhythmic electrical modulation system (FREMS) has been recently reported to improve the claudication distance (CD) and micro-circulation thus mimicking the effects of exercise ${ }^{(5)}$.

On the other hand the Pulsed low frequency electromagnetic field (PLFEM) is an interesting therapeutic procedure. It achieved significant improvements when utilized in multiple peripheral vascular and musculoskeletal disorders treatment ${ }^{(5)}$. The therapy of PEMF is achieved by altering biological and physiological systems via low energy and nonionizing electromagnetic fields. PEMF therapy was originally used clinically to manage osteoarthritisrelated pain and stiffness and to augment bone healing. 
In addition, recent research has explored the beneficial therapeutic effect of PEMF on microvasculature and circulation. More recently, research interests have expanded their focus to additional mechanisms and syndromes, including a focus on the effects of PEMF on peripheral vascular function and blood flow ${ }^{(6)}$.

Pulsed electromagnetic therapy can successfully help improvement of conditions and diseases, including those associated with vasoconstriction (e.g., coronary vasospasm, elevated systemic vascular resistance and hypertension), thrombosis due to platelet aggregation and adhesion to vascular endothelium, inflammation due to up regulation of leukocyte and endothelial adhesion molecules, vascular hypertrophy and stenosis, and consequently hypertension ${ }^{(\boldsymbol{6})}$.

Therefore, the purpose of the present study was to compare between the effects of two different modalities: Interferential electrical stimulation and pulsed electromagnetic field on intermittent claudication.

\section{SUBJECTS AND METHODS}

Randomized controlled study conducted in Ahmed Maher Teaching Hospital in Cairo at the period between September 2019 and March 2020. The whole procedure was explained for every patient. Each patient signed informed consent before beginning of the study to insure complete satisfaction.

\section{Subjects:}

Forty five patients of both sexes ( 28 males, 17 females) suffering from intermittent claudication due to atherosclerosis (Fontain stage II PAD, intermittent claudication) participated in this study. They were, assigned randomly into three groups, group A (GA), group B (GB) and control group (GC) after consenting to participate and fulfilling the inclusion criteria. GA received pulsed electromagnetic field in addition to conventional physiotherapy exercises in the form of heel raise exercise and calf stretching exercise plus their traditional medical treatment. GB received interferential current field in addition to conventional physiotherapy exercises in the form of heel raise exercise and calf stretching exercise plus their traditional medical treatment. Control group (GC) received their medical treatment only.

Inclusion criteria: (a) Patients with mild to moderate intermittent claudication (according to Rutherford classification) ${ }^{(7)}$. (b) Both sex with ages ranged from 50 to 60 years. (c) All patients enrolled to the study gave their informed consent. (d) The patients were suffering from spasmodic pain in the calf, thigh, or buttocks that limited walking ability and that was relieved by rest within 10 minutes. (e) Patients with Ankle Brachial Index (ABI) ranged between 0.9 to 0.5.

Exclusion criteria: (a) Fontain stage (I) PAD (asymptomatic disease) and Fontain stage (III) PAD (leg pain at rest). (b) Patients with severe symptomatic coronary artery disease and cerebrovascular disease.

(c) Patients with poorly controlled blood pressure.

(d) Those who underwent vascular operations or angioplasty within the previous year. (e) Dementia or physical/mental incapacity to perform study requirements. (f) Musculoskeletal disorders in lower limbs such as recent fracture, hip dislocation, nerve root compression, spinal stenosis, symptomatic Baker's cyst and chronic compartment syndrome that may mimic the symptoms of intermittent claudication. (g) Active cancer, renal disease, or liver disease. (h) Patients with cardiac pacemaker.

\section{Ethical approval and written informed consent:} An approval of the study was obtained from Cairo University academic and ethical committee. Every patient signed an informed written consent for acceptance of the operation.

\section{Assessment instrument:}

1- Mercury sphygmomanometer: Speidel, Keller, rninia Tur 300 (made in Germany) and stethoscope (Littman, classic II, made in USA), hand held Doppler (Bistos Hi-dop BT200V, 5 MHZ probe) used to measure the systole of ankle and brachial arteries pressure to determine ankle brachial pressure index.

2- Electric treadmill (Electrical treadmill bodywork sport 1500 made in USA): was used to assess absolute claudication distance (ACD) and peak walking time (PWT).

3- Walking impairment questionnaire (WIQ): it consisted of 3 primary categories (distance score, speed score and walking impaired symptoms).

\section{Treatment instruments:}

1- Pulsed electromagnetic field therapy (PEMF): electromagnetic unit ASA magnetic field (Automatic PMT Quattro pro): It consisted of an appliance, motorized bed and solenoids. The appliance was connected to electrical mains supplying $230 \mathrm{~V}$ at a frequency of 50 or $60 \mathrm{~Hz}$ with earth connection. It generated pulsed magnetic field up to $100 \mathrm{~Hz}$ and intensity varied according to the type of solenoid. It was used in the treatment of patient in group "A"

2- Interferential electrical stimulator: BTL-4620 PROFESSIONAL this portable electrotherapy unit with 2 channels has graphic display. It was used in the treatment of patient in group "B".

\section{Procedures:}

\section{A) Assessment procedures:}

Ankle brachial pressure index (ABPI) was measured, by physiotherapist for all patients from supine lying position, using cuff of $12 \mathrm{~cm}$ wide that was placed just around the ankle. Sufficient gel was used on the artery and use The Doppler probe which emits a 
high frequency signal to detect arterial flow. The device converts the frequency shift into audible signal. The cuff was inflated until the pulse in the artery ceases. The blood pressure cuff is then slowly deflated. When the artery's pulse is re-detected through the Doppler the pressure in the cuff at that moment indicates the systolic pressure of that artery. Measurement was made for both the dorsalis pedis and posterior tibial and the highest value was used. For brachial systolic measurement, the pressure was measured from both upper limbs. The higher systolic reading of the left and right arm brachial artery is generally used in the assessment. ABPI was obtained by dividing ankle systolic pressure over brachial systolic pressure according to the following equation ${ }^{(\mathbf{8})}$ :

Ankle brachial pressure index $=$

Ankle systolic pressure ( $\mathrm{mmHg}$ )

$\overline{\text { Brachial systolic pressure }(\mathrm{mm} \mathrm{Hg})}$

\section{Graded treadmill exercise testing:}

Patients started walking on treadmill with $2 \mathrm{mph}$, 0 grade. With gradually increasing the inclination (2\% increase every 2 minutes) until maximal claudication pain force cessation of the exercise. The perception of claudication pain severity was ascertained every 30 seconds using a perceived pain scale from 1 to $5.1=$ no pain and the longest possible walking distance reached by the patient before the appearance of intolerable pain is the absolute claudication distance. The walking time at which ambulation could not continue due to maximum pain was defined as Peak walking time ${ }^{(9,10)}$.

\section{Walking Impairment Questionnaire (WIQ):}

Patients were asked to rate the degree of difficulty of various activities with responses ranging from 0 (unable) to 4 (none). Walking distance questions range from walking indoors to walking 1500 feet. Walking speed questions range from walking 1 block slowly to jogging 1 block. Questions within each category are then weighted based on the degree of difficulty, according to the approximate number of feet or miles per hour for the distance and speed scores respectively.

Scores are then divided by the maximum number of points and presented on a scale of $0 \%$ to $100 \%$, where $0 \%$ represents the lowest possible score (i.e., answering "unable" for all questions in that category) and $100 \%$ represents the highest possible score (i.e., indicating "none" with regard to difficulty for all questions in that category) ${ }^{(11)}$.

\section{B) Treatment procedures:}

Patients in group (A): received a pulsed electromagnetic field with low frequency $(10-50 \mathrm{~Hz})$ and low intensity $(10 \mathrm{mT})$ that was applied on the calf of ischemic extremity by solenoid with 30 min duration for 24 sessions (3 sessions per week). Patients also received heel raise exercise and calf stretching exercise while they were on their medical treatment. Patients in group (B) received interferential electrical stimulation and received treatment from side lying position with electrodes placed along the ischemic extremity with $10-100 \mathrm{~Hz}$ frequency and 20 minutes duration for 24 sessions ( 3 sessions per week). Patients also received heel raise exercise and calf stretching exercise while they were on their medical treatment.

\section{Statistical Analysis}

Descriptive statistics and ANOVA test were conducted for comparison of age, weight, height and BMI between the three groups. Chi- square test was used for comparison of sex distribution between the three groups. Normal distribution of data was checked using the Shapiro-Wilk test for all variables. Levene's test for homogeneity of variances was conducted to test the homogeneity between groups.

ANOVA was performed to compare absolute claudication distance, peak walking time, ankle brachial pressure index and walking impairment questionnaire at pre and post between the three groups. Post-hoc tests using the Tukey test were carried out for subsequent multiple comparison. Paired t test was carried out for comparison between pre and post treatment mean values in each group. The level of significance for all statistical tests was set at $p<0.05$. All statistical analysis was conducted through the statistical package for social studies (SPSS) version 25 for windows (IBM SPSS, Chicago, IL, USA).

\section{RESULTS}

Table (1): Basic characteristics of participants

\begin{tabular}{|c|c|c|c|c|c|}
\hline & $\begin{array}{c}\text { Group A } \\
\text { mean } \pm \text { SD }\end{array}$ & $\begin{array}{c}\text { Group B } \\
\text { mean } \pm \text { SD }\end{array}$ & $\begin{array}{c}\text { Group C } \\
\text { mean } \pm \text { SD }\end{array}$ & F- value & p-value \\
\hline Age (vears) & $54.46 \pm 3.88$ & $55.06 \pm 2.65$ & $55.86 \pm 3.54$ & 0.63 & 0.53 \\
\hline Weight (kg) & $80.7 \pm 7.27$ & $80.5 \pm 6.65$ & $81.1 \pm 6.61$ & 0.03 & 0.97 \\
\hline Height (cm) & $168.43 \pm 4.45$ & $167.83 \pm 6.06$ & $168.4 \pm 6.25$ & 0.05 & 0.94 \\
\hline BMI $\left(\mathrm{kg} / \mathrm{m}^{2}\right)$ & $28.46 \pm 2.48$ & $28.65 \pm 2.86$ & $28.57 \pm 1.37$ & 0.02 & 0.97 \\
\hline \multicolumn{6}{|l|}{$\operatorname{Sex}, \mathrm{n}(\%)$} \\
\hline Females & $6(40 \%)$ & $4(27 \%)$ & $7(47 \%)$ & $\left(\begin{array}{llll}\gamma^{2} & 1 & 32\end{array}\right)$ & 051 \\
\hline Males & $9(60 \%)$ & $11(73 \%)$ & $8(53 \%)$ & & 0.51 \\
\hline
\end{tabular}

$\mathrm{SD}$, standard deviation; $\chi 2$, Chi squared value; $p$-value, level of significance 
Forty-five patients with intermittent claudication participated in this study. Subjects were subdivided into three groups, fifteen in each group. Data obtained from the three groups pre and post treatment regarding absolute claudication distance (ACD), peak walking time (PWT), ankle brachial pressure index (ABPI) and walking impairment questionnaire (WIQ) were statistically analyzed and compared. Comparing the general characteristics of the subjects of the three groups revealed that there was no significant difference between groups in the mean age, weight, height and BMI (p > 0.05) (Table 1).

Effect of treatment on absolute claudication distance, peak walking time, ankle brachial pressure index and walking impairment questionnaire:

Within group comparison:

Absolute claudication distance, peak walking time, ankle brachial pressure index:

There was a significant increase in the absolute claudication distance and peak walking time in groups $\mathrm{A}, \mathrm{B}$ and $\mathrm{C}$ post-treatment compared to pretreatment ( $\mathrm{p}$ $<0.001)$. There was a significant increase in the ankle brachial pressure index in group A post-treatment compared to pretreatment $(\mathrm{p}=0.02)$, while there was no significant difference between pre- and posttreatment in groups B and C ( $p>0.05)$ (Table 2, Figures $1-3)$.

\section{Walking impairment questionnaire:}

There was a significant increase in the distance, speed and symptoms scores in groups A, B and C post treatment compared to pretreatment $(\mathrm{p}<0.001)$ (Table 3, Figure 4).

Between group comparison:

Absolute claudication distance, peak walking time and ankle brachial pressure index:

Pretreatment revealed a non-significant difference in all parameters $(\mathrm{p}>0.05)$. Post treatment revealed a significant increase in absolute claudication distance and peak walking time of group A compared to that of group B and group $\mathrm{C}(\mathrm{p}<0.05)$ and a significant increase in group $\mathrm{B}$ compared to that of group $C(p<0.05)$. There was no significant difference in the ankle brachial pressure index between the three groups post treatment $(\mathrm{p}>0.05)$ (Table 2, Figures 1-3).

\section{Walking impairment questionnaire:}

There was no significant difference between groups in walking impairment questionnaire pretreatment $(\mathrm{p}>0.05)$. There was a significant increase in distance, speed and symptoms scores of group A compared to that of group B and group $\mathrm{C}$ ( $\mathrm{p}<$ 0.05 ) and a significant increase in group $B$ compared to that of group $C(p<0.05)$ (Table 3, Figure 4).

Table (2): Mean absolute claudication distance, peak walking time and ankle brachial pressure index pre and post treatment of groups A, B and C.

\begin{tabular}{|c|c|c|c|c|c|c|}
\hline & \multirow{2}{*}{$\begin{array}{r}\text { Group A } \\
\text { mean } \pm \text { SD }\end{array}$} & \multirow{2}{*}{$\begin{array}{c}\text { Group B } \\
\text { mean } \pm \text { SD }\end{array}$} & \multirow{2}{*}{$\begin{array}{c}\text { Group C } \\
\text { mean } \pm \text { SD }\end{array}$} & \multicolumn{3}{|c|}{ p-value } \\
\hline & & & & A vs B & A vs C & B vs C \\
\hline \multicolumn{7}{|c|}{ Absolute claudication distance $(m)$} \\
\hline Pre-treatment & $228.46 \pm 23.53$ & $226.66 \pm 27.6$ & $225.73 \pm 22.68$ & 0.97 & 0.95 & 0.99 \\
\hline Post treatment & $356.26 \pm 48.67$ & $313.93 \pm 43.97$ & $274.06 \pm 32.02$ & 0.02 & 0.001 & 0.03 \\
\hline MD & -127.8 & -87.27 & -48.33 & & & \\
\hline$\%$ of change & 55.94 & 38.5 & 21.41 & & & \\
\hline \multirow[t]{2}{*}{ t-value } & -11.14 & -10.56 & -6.78 & & & \\
\hline & $p=0.001$ & $p=0.001$ & $p=0.001$ & & & \\
\hline \multicolumn{7}{|c|}{ Peak walking time (min) } \\
\hline Pre-treatment & $6.13 \pm 1.44$ & $6.08 \pm 1.64$ & $6.17 \pm 1.66$ & 0.99 & 0.99 & 0.98 \\
\hline Post treatment & $10.08 \pm 1.85$ & $8.37 \pm 1.53$ & $6.85 \pm 1.54$ & 0.01 & 0.001 & 0.04 \\
\hline MD & -3.95 & -2.29 & -0.68 & & & \\
\hline$\%$ of change & 64.44 & 37.66 & 11.02 & & & \\
\hline \multirow[t]{2}{*}{ t-value } & -12.89 & -14.72 & -8.59 & & & \\
\hline & $p=0.001$ & $p=0.001$ & $p=0.001$ & & & \\
\hline \multicolumn{7}{|c|}{ Ankle brachial pressure index } \\
\hline Pre treatment & $0.77 \pm 0.11$ & $0.74 \pm 0.08$ & $0.76 \pm 0.11$ & 0.69 & 0.98 & 0.78 \\
\hline Post treatment & $0.8 \pm 0.12$ & $0.75 \pm 0.09$ & $0.77 \pm 0.11$ & 0.42 & 0.8 & 0.8 \\
\hline MD & -0.03 & -0.01 & -0.01 & & & \\
\hline$\%$ of change & 3.9 & 1.35 & 1.32 & & & \\
\hline t-value & -2.47 & -1.47 & -1.56 & & & \\
\hline & $p=0.02$ & $p=0.16$ & $p=0.13$ & & & \\
\hline
\end{tabular}

$\mathrm{SD}$, Standard deviation; MD, Mean difference; p-value, Level of significance 
Table (3): Mean walking impairment questionnaire pre- and post-treatment of group A, B and C:

\begin{tabular}{|c|c|c|c|c|c|c|}
\hline \multirow{2}{*}{$\begin{array}{c}\text { Walking impairment } \\
\text { questionnaire }(\%)\end{array}$} & \multirow{2}{*}{$\begin{array}{c}\text { Group A } \\
\text { mean } \pm \text { SD }\end{array}$} & \multirow{2}{*}{$\frac{\text { Group B }}{\text { mean } \pm \text { SD }}$} & \multirow{2}{*}{$\begin{array}{c}\text { Group C } \\
\text { mean } \pm \text { SD }\end{array}$} & \multicolumn{3}{|c|}{ p-value } \\
\hline & & & & A vs B & A vs C & B vs C \\
\hline \multicolumn{7}{|l|}{ Distance score } \\
\hline Pre treatment & $18.09 \pm 3.12$ & $18.43 \pm 3.01$ & $17.58 \pm 2.46$ & 0.94 & 0.88 & 0.7 \\
\hline Post treatment & $34.05 \pm 6.81$ & $28.91 \pm 4.88$ & $23.56 \pm 4.05$ & 0.03 & 0.001 & 0.02 \\
\hline MD & -15.96 & -10.48 & -5.98 & & & \\
\hline$\%$ of change & 88.23 & 56.86 & 34.02 & & & \\
\hline \multirow[t]{2}{*}{ t-value } & -8.61 & -9.96 & -5.34 & & & \\
\hline & $p=0.001$ & $p=0.001$ & $p=0.001$ & & & \\
\hline \multicolumn{7}{|l|}{ Speed score } \\
\hline Pre treatment & $13.7 \pm 2.33$ & $13.94 \pm 1.73$ & $13.83 \pm 2.27$ & 0.94 & 0.98 & 0.98 \\
\hline Post treatment & $23.84 \pm 5.87$ & $19.93 \pm 2.95$ & $15.88 \pm 2.87$ & 0.03 & 0.001 & 0.02 \\
\hline MD & -10.14 & -6 & -2.05 & & & \\
\hline$\%$ of change & 74.01 & 43.04 & 14.82 & & & \\
\hline \multirow[t]{2}{*}{ t-value } & -7.87 & -7.02 & -3.6 & & & \\
\hline & $p=0.001$ & $p=0.001$ & $p=0.003$ & & & \\
\hline \multicolumn{7}{|l|}{ Symptoms score } \\
\hline Pre treatment & $19.6 \pm 1.84$ & $20.4 \pm 1.55$ & $19.93 \pm 1.38$ & 0.36 & 0.83 & 0.7 \\
\hline Post treatment & $24 \pm 1.7$ & $22.2 \pm 0.94$ & $20.86 \pm 1.35$ & 0.002 & 0.001 & 0.02 \\
\hline MD & -4.4 & -1.8 & -0.93 & & & \\
\hline$\%$ of change & 22.45 & 8.82 & 4.67 & & & \\
\hline \multirow[t]{2}{*}{ t-value } & -10.68 & -6.44 & -7.89 & & & \\
\hline & $p=0.001$ & $p=0.001$ & $p=0.001$ & & & \\
\hline
\end{tabular}

$\mathrm{SD}$, Standard deviation; MD, Mean difference; $\mathrm{p}$-value, Level of significance

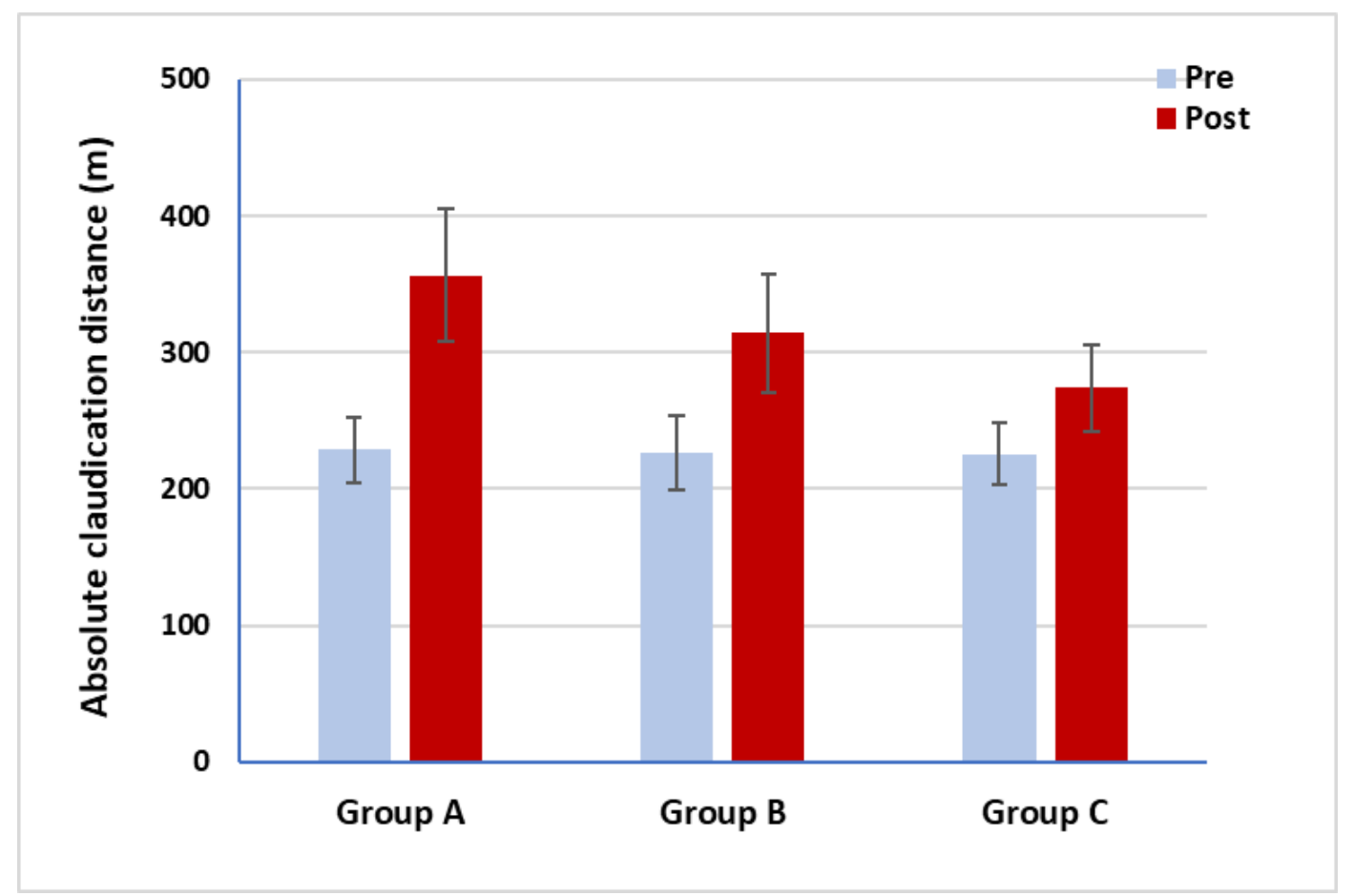

Figure (1): Mean absolute claudication distance pre- and post-treatment of groups A, B and C. 


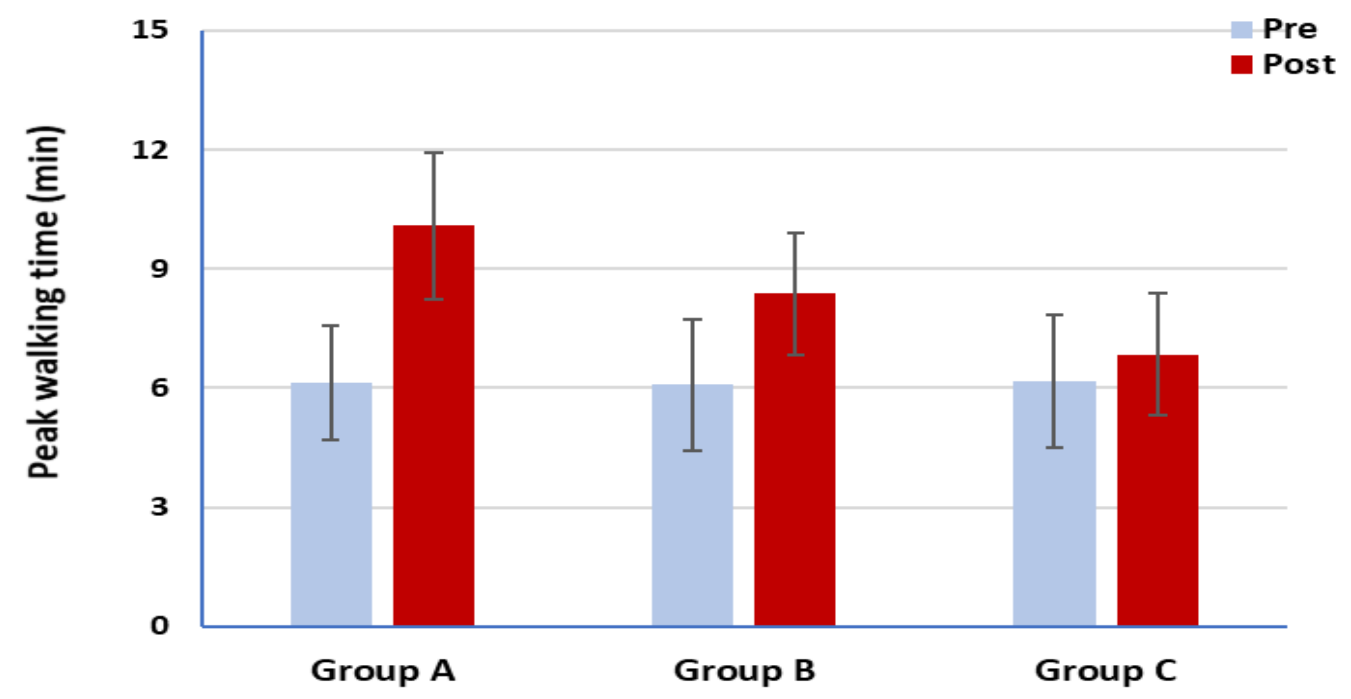

Figure (2): Mean peak walking time pre- and post-treatment of groups A, B and C.

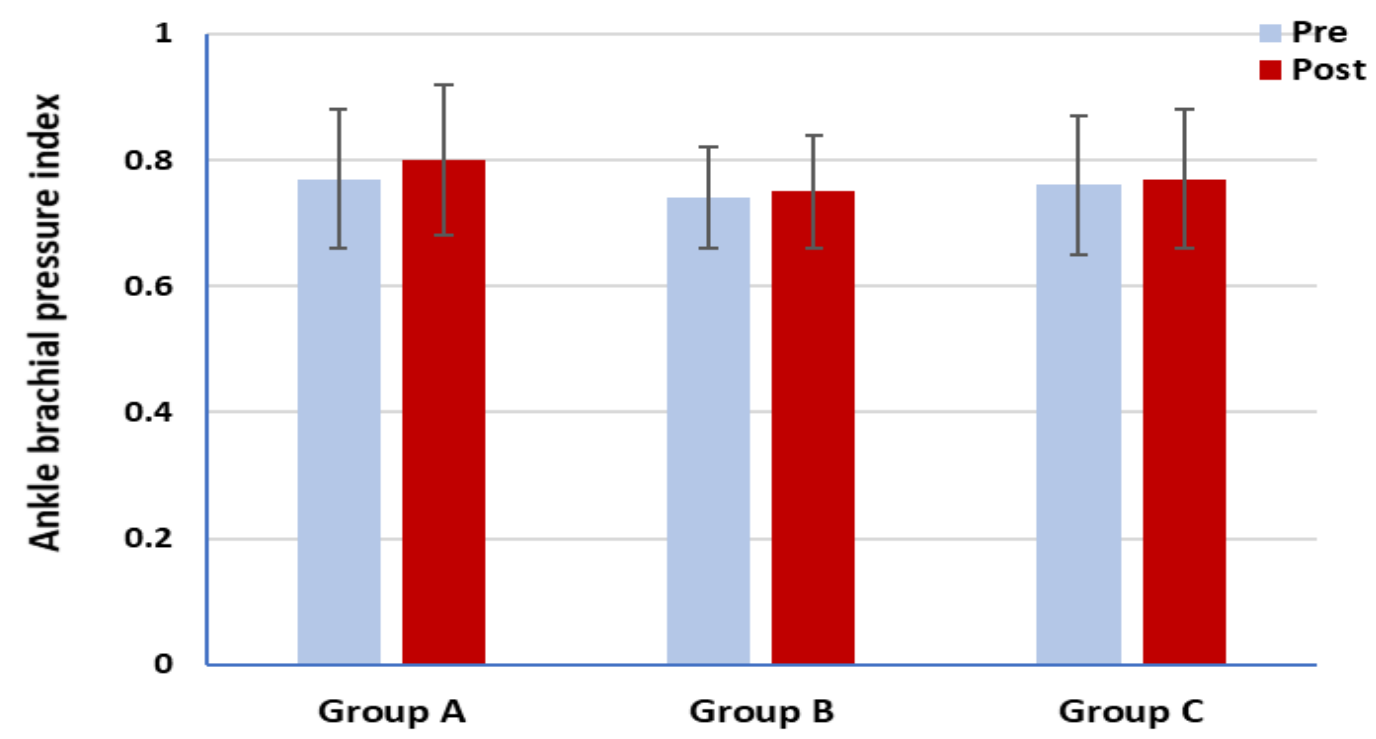

Figure (3): Mean ankle brachial pressure index pre- and post-treatment of groups A, B and C.

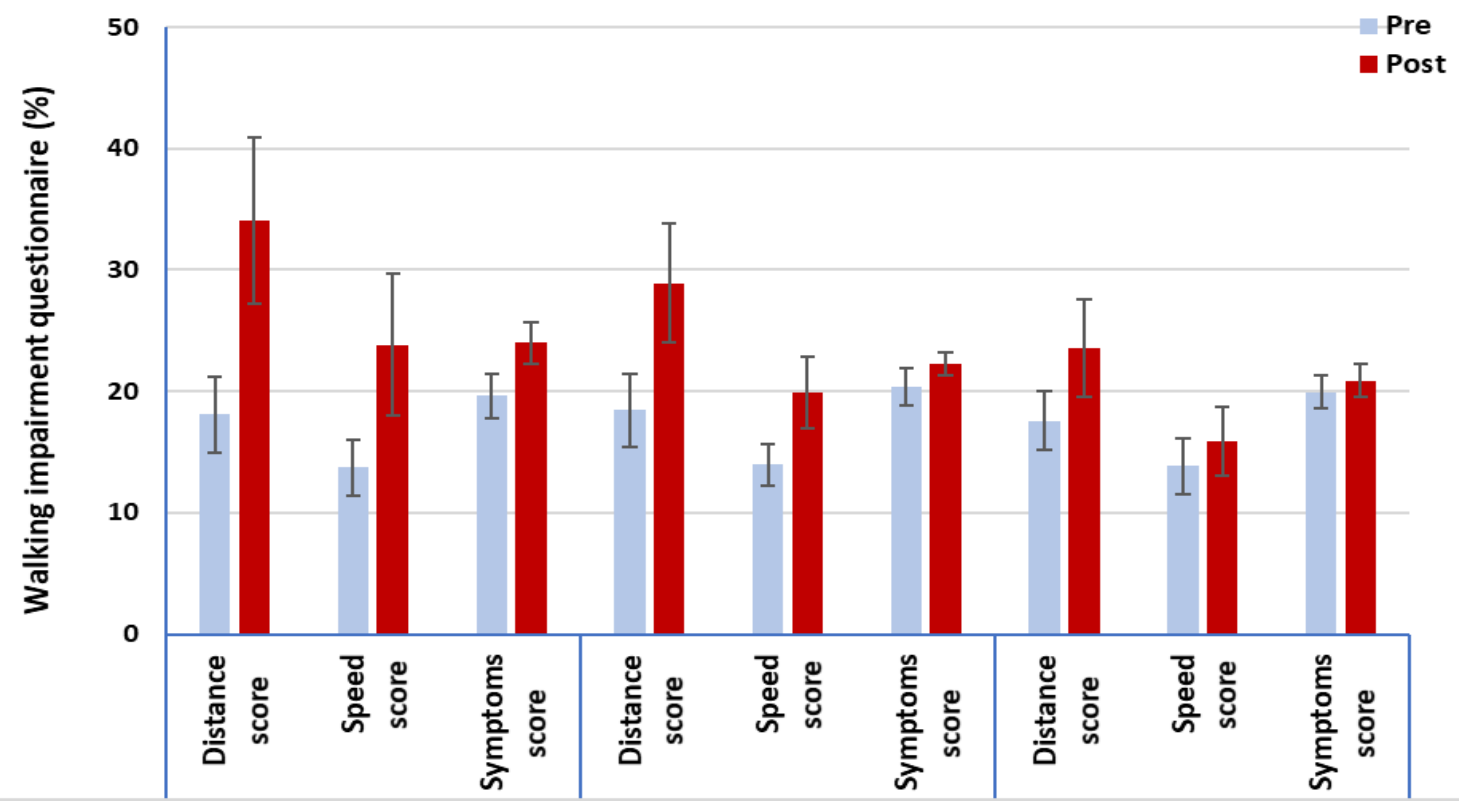

Figure (4): Mean walking impairment questionnaire pre- and post-treatment of groups A, B and C. 


\section{DISCUSSION}

The aim of this study was to investigate and compare between the effect of interferential electrical stimulation (IFT) and pulsed electromagnetic field in management of intermittent claudication in peripheral artery disease.

In the present study, the outcome measurements were considered to increase the pain free and maximal walking distance, improve walking economy and to be measured objectively through ABPI, graded treadmill exercise testing the study results showed the following significant improvement in pulsed electromagnetic group (A) compared to interferential current (B) and control group (C) as following: significant improvement in ACD and PWT, significant improvement in walking impairment questionnaire distance and speed score and impaired symptoms score.

The improvement of absolute claudication distance coincides with the result of Miroslav et al. ${ }^{(5)}$ who conducted a randomized study included 47 patients with peripheral arterial occlusive disease manifested by intermittent claudications associated with ankle-brachial indexes values ranging from 0.5 to 0.9. Patients from the first group were treated with medicamentous therapy, walking exercises beyond the pain threshold, dynamic low-burden kinesi exercises and electrotherapeutic ageneses (interference therapy and electromagnetic field). The second group of patients was treated with "conventional" medicamentous therapy and walking exercises. They found an increase in the claudication distance interval in the PEMF and interfrential current group and there was no significant increase in ankle- brachial indexes values in both groups of patients. Also Rikk et al. ${ }^{(12)}$ demonstrated the effects of pulsating electromagnetic field (PEMF) therapy sessions on the changes in peripheral cardiovascular function in a group of aging adults after 12 weeks of treatment. The results showed statistically significant reductions in systolic and pulse

blood pressure. These findings suggest that PEMF treatment might be linked to improvements in peripheral resistance or circulation and this in itself is a significant and positive impact on the overall patient's well-being (among other things, i.e. improved walking distance), which directly enhances a greater motivation for movement.

The results of the present study could be explained by the findings of Bragin et al. ${ }^{(13)}$ who reported that thirty minutes of PEMF treatment induced cerebral arteriolar dilatation leading to an increase in micro vascular blood flow and tissue oxygenation that persisted for at least 3 hours. The effects of PEMF were mediated by NO, the most important vasodilator and a part from the improvement of microcirculation and enhancement of transport function of blood.

In addition, Tepper et $\boldsymbol{\text { al }} .{ }^{(14)}$ demonstrated that delivery of PEMF at low doses, identical to that currently in clinical use, significantly increased endothelial cell proliferation and tubulization, processes important for vessel formation. The ability of PEMF to increase cellular proliferation was unique to endothelial cells. This suggests that endothelial cells are the primary target for PEMF stimulation, releasing protein in a paracrine fashion to induce changes in neighboring cells and up-regulate angiogenesis.

The improved walking distance seen also in group (B) who received interferential stimulation were explained by Cho et al. ${ }^{\left({ }^{(15)}\right.}$ who reported that a $100-\mathrm{HZ}$ electrical stimulation affects the balance of central and local factors at a systemic level and that may be attributed to the functional recovery of tissues by actively stimulating them and controlling associated pain, relaxing contracted muscles by inducing vasodilatation and increasing blood flow. Interferential current (IFC) is thought to exert its analgesic effects via the direct stimulation of the muscle fibers rather than the peripheral nerves, thus promoting the therapeutic process. Interesting findings were reported by Tsang and Green ${ }^{(16)}$ in a study where patients with P.A.D were submitted to Electrical stimulation of flexor muscles in the ankle for 20 minutes 3 times a day for 4 weeks in total. Authors reported a significant increase in muscle functional performance. However, after therapy cessation, performance of the muscles recessed to its initial levels.

So, interferential electrical stimulation alone was also effective but its impact was less than PEMF.

\section{CONCLUSION}

The results of this study support the good effect of pulsed electromagnetic field on patient with intermittent claudication pain from peripheral arterial occlusive disease. Pulsed electromagnetic field will improve the patient's functional level and the capacity for independent living by increasing significantly the absolute claudication distance, peak walking time and ankle brachial pressure index as well as walking impairment questionnaire.

\section{REFERENCES}

1. Aronow WS (2012): Peripheral arterial disease of the lower extremities. J of Arch Med Sci., 8 (2): 375-388.

2. Meru AV, Mittra S, Thyagarajan B et al. (2006): Intermittent claudication: An overview. $\mathrm{J}$ of Atherosclerosis, 187: 221-237.

3. McDermott MM (2015): Lower extremity manifestations of peripheral artery disease:the pathophysiology and functional implications of leg ischemia. J of Circ Res., 116: 1540-1550.

4. Cassar K (2006): Intermittent claudication. British Med J., 333: 100-102. 
5. Miroslav M, Koncar I, Dragas M (2015): The role of kinesitherapy and electrotherapeutic procedures in non-operative management of patients with intermittent claudications. Sage Journals, 24 (3):1-8.

6. Kim CH, Wheatley CM, Stewart GM et al. (2019): The impact of pulsed electromagnetic field therapy on blood pressure and circulating nitric oxide levels: a double blind, randomized study in subjects with metabolic syndrome. Blood Pressure Journal, 29 (1): 47-54.

7. Rutherford RB, Baker JD, Ernst C (1997): Recommended standards for reports dealing with lower extremity ischemia: revised version. J Vasc Surg., 26: 517-38.

8. Al-Qaisi M, Nott DM, King DH et al. (2009): Ankle brachial pressure index (ABPI): An update for practitioners. Vascular Health and Risk Management, 5: 833-41.

9. Gardner AW, Montgomery PS, Donald E et al. (2012): Optimal exercise program length for patients with claudication: a randomized controlled trial. J Vasc Surg., 55 (5): 1346-1354.

10. Gardner AW (1997): Reliability of transcutaneous oximeter electrode heating power during exercise in patients with intermittent claudication. Angiology, 48 (3): 229-35.
11. Nead KT, Zhou M, Caceres RD et al. (2013): Walking impairement questionnaire improves mortality risk predection models in a high risk cohort independent ofperipheral artery disease status. AHA Journals, 3: 256-261.

12. Rikk J, Finn KJ, Liziczai I et al. (2013): Influence of pulsing electromagnetic field therapy on resting blood pressure in aging adults. $\mathbf{J}$ of Electromagnetic Biology and Medicine, 32 (2): 165-172.

13. Bragin DE, Statom GL, Hagberg S et al. (2015): Increases in microvascular perfusion and tissue oxygenation via pulsed electromagnetic fields in the healthy rat brain. J Neurosurg., 122 (5): 989-1251.

14. Tepper OM, Callaghan MJ, Chang EI et al. (2004): Electromagnetic fields increase in vitro and in vivo angiogenesis through endothelial release of FGF2. The FASEB Journal, 18 (11): 1169-1323.

15. Cho SH (2019): Frequency and Intensity of Electrical Stimulation of Human Sympathetic Ganglia Affect Heart RateVariability and Pain Threshold. Applied Sciences, 9 (21): 4490.

16. Tsang GMA, Green MA (1994): Chronic Muscle Stimulation Improves Ischaemic Muscle Performance in Patients with Peripheral Vascular Disease. Eur J of Vasc Surg., 8: 419-422. 